\title{
L'alcool : une drogue licite aux conséquences bucco-dentaires non négligeables
}

\author{
Alcohol: a licenced drug \\ with significant oral consequences
}

\section{MOTS-CLEFS :}

- Drogue licite, alcool, toxicomanie, addiction, effets bucco-dentaires.

\section{KEYWORDS:}

- Licit drug, alcohol, dependence, mouth diseases.

\section{Résumé}

Si le terme de toxicomanie est souvent associé à la consommation de substances illicites (héroïne, cocaïne), Il faut aujourd'hui l'étendre à des produits licites comme l'alcool. La toxicomanie à l'alcool est une notion complexe qui fait intervenir un individu dans une société et une substance psychoactive source de plaisir. Les conséquences d'une consommation abusive sur le milieu buccal existent : xérostomie, halitose, atteintes carieuses et parodontales, érosion et pertes dentaires, risque de non ostéo-intégration implantaire, péri implantite, lésions précancéreuses et cancéreuses, enfin traumatismes divers, résultats d'état d'ébriété. Dans cette optique, le rôle du chirurgien-dentiste est fondamental, à la fois pour faire prendre conscience de la réalité de l'addiction mais aussi dans la prévention et le dépistage des pathologies buccales induites par cette substance toxique.

\section{Abstract}

Usually the term toxicomania is often associated with the consumption of illegal substances (heroin, cocaine), but nowadarys we have to extend it to legal products such as alcohol. Alcohol addiction is a complex notion which relates to an individual in a society and a pleasure giving psychoactive substance. Consequences of an excessive consumption on the buccal environment exist: xerostomia, halitosis, dental caries, periodontal disease, erosion and tooth loss, failure of the implant to osseointegrate correctly, peri-implantitis, precancerous and cancerous lesions, and finally diverse dental traumas, as results of drunkenness. In this perspective, the role of the dentist is fundamental, firstly to allow the patient to become aware of the addiction but also to prevent and screen the oral diseases induced by this toxic substance.
AOS 2013;262:24-30

DOI: $10.1051 / \mathrm{aos} / 2013205$

(C) EDP Sciences 2013

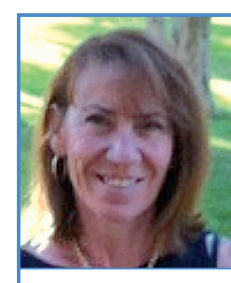

- Catherine PESCI-BARDON, Docteur en chirurgie dentaire, Docteur de l'université Nice-Sophia Antipolis,

Praticien hospitalier,

Pôle odontologie, Hôpital Saint-Roch, Centre hospitalier universitaire de Nice, 5, rue Pierre Dévoluy, BP 1319, o6006 Nice cedex 01.

Laboratoire de Santé buccale et vieillissement, faculté d'odontologie, université de Nice-Sophia Antipolis, o6357 Nice cedex 4, France.

Isabelle PRECCHEUR, Docteur en chirurgie dentaire,

Professeur de l'université Nice-Sophia Antipolis,

Praticien hospitalier, Pôle odontologie, Hôpital Saint-Roch,Centre hospitalier universitaire de Nice, 5, rue Pierre Dévoluy, BP 1319, 06006 Nice cedex 01.

Laboratoire de Santé buccale et vieillissement, faculté d'odontologie, université de Nice-Sophia Antipolis, o6357 Nice cedex 4, France. 
$\mathrm{D}$ e tout temps, les individus dans les sociétés ont consommé des substances psychoactives (substances d'origine naturelle ou synthétique qui agissent sur le psychisme en modifiant l'activité mentale, les sensations, l'humeur et le comportement). Ces produits aident le sujet à se sentir moins vulnérable, plus sociable, plus serein et favorisent un certain sentiment de bien-être. Ils modifient les sensations et les rapports avec le monde extérieur, voire le rapport à l'individu lui-même. Toutes les drogues licites ou illicites sont psychoactives, leur utilisation pouvant conduire à l'accoutumance et à la dépendance donc à la toxicomanie.

Les drogues illicites (héroïne, cocaïne, cannabis...) sont les drogues interdites par le code pénal qui en sanctionne la production, la détention et la vente, leur consommation est également réprimandée. Tabac et alcool sont considérés comme des drogues licites dont la vente est contrôlée et l'utilisation dans les lieux publics strictement réglementée [1]. Depuis une vingtaine d'années, des campagnes de prévention mettent en garde la population sur la consommation de certains produits considérés autrefois comme relativement anodins, c'est le cas de l'alcool dont les effets néfastes sur la santé sont mis en avant, même si concernant le produit et les comportements compulsifs qu'il peut engendrer, il ne soit pas perçu comme une drogue.

Le chirurgien-dentiste hospitalier est confronté quotidiennement au patient sous l'emprise de l'alcool, qu'il s'agisse d'un alcoolisme aigu qui se manifeste par une consommation occasionnelle importante (appelée encore binge drinking) sans phénomène de dépendance ou d'un alcoolisme chronique qui se traduit par une consommation répétée quotidienne au-delà des seuils de toxicité (2 à 3 verres standards par jour) et qui n'a pas forcément pour objectif l'ivresse majeure, mais qui provoque une dépendance. Le tableau clinique est souvent sous-tendu par l'angoisse et la dépression [2].

\section{MODALITÉS DE CONSOMMATION}

Dans l'espace festif, depuis plusieurs années, le développement des binge drinking est de plus en plus rapporté. Cette pratique consiste à absorber de grandes quantités d'alcool dans un délai de temps réduit, de manière à accéder rapidement à un état d'ivresse. Il semble en effet que, quel que soit le lieu festif (clubs, discothèques ou free parties), la consommation d'alcool soit de plus en plus souvent orientée vers la recherche d'effet de « défonce ». Les acteurs sanitaires et répressifs font état d'une banalisation de ces pratiques d'usage massif qui tendent à se régulariser, généralement hebdomadaires, elles sont décrites dans des populations relativement jeunes et notamment au cours des fêtes étudiantes. D'autre part, il faut savoir qu'en France, le risque d'alcoolisation excessive, ponctuel ou chronique, concerne surtout les hommes : plus de quatre hommes sur dix contre une femme sur dix. Entre 25 et 64 ans, il touche un homme sur deux. Ces comportements sont moins fréquents chez les personnes vivant au sein d'une famille, sauf lorsque l'un des membres présente un usage à risque. Les liens entre le risque d'alcoolisation excessive et les catégories socio-économiques sont contrastés. Chez les femmes, le risque n'est patent que pour les cadres alors que, chez les hommes, il touche aussi bien les ouvriers que les cadres, mais moins fréquemment les employés. Le risque est plus souvent chronique que ponctuel chez les personnes ayant connu des épisodes de précarité au cours de leur vie, chez les hommes exerçant une profession intermédiaire, chez les artisans, commerçants ou chefs d'entreprise, ainsi que chez les hommes ayant des revenus faibles. La non-consommation, quant à elle, se concentre dans les catégories les moins aisées [3,4]. Cependant, les femmes qui accèdent de plus en plus à l'égalité avec les hommes, en imitent les prérogatives, même nocives : fumer, boire, stresser au travail. Le féminisme conquiert, et recopie, scories incluses [5].

\section{LIMITES DE CONSOMMATION}

Une unité d'alcool est équivalente à un verre standard (de vin, bière, pastis, planteur...) et égale 10 grammes d'alcool (fig. 1). Pour l'OMS, une consommation modérée d'alcool se situe à :

1 moins de 2 verres par jour pour une femme (soit moins de 14 verres par semaine);

moins de 3 verres par jour pour un homme (soit moins de 21 verres par semaine);

pas plus de 4 verres par occasion;

un jour sans alcool par semaine ;

$\checkmark$ pas d'alcool dans certaines circonstances (grossesse, conduite de véhicule, prise de certains médicaments tels que psychotropes, métiers ou sports dangereux, certaines pathologies...).

L'usage est à risque si la consommation est supérieure aux seuils préconisés par l'OMS.
Fig. 1 :

Un verre standard égale 10 grammes d'alcool.

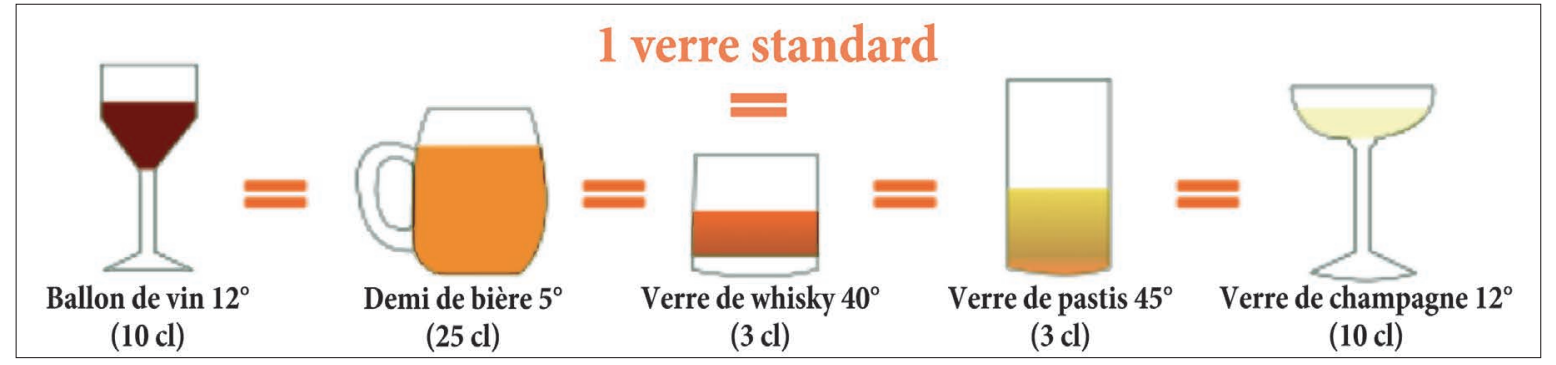


L'usage est nocif ou abusif si des dommages avérés apparaissent dans les domaines sanitaires ou sociaux, pour le sujet ou pour son entourage, sans qu'il y ait dépendance à l'alcool. On parle d'usage avec dépendance s'il y a perte de maîtrise de la consommation d'alcool, «perte de la liberté de s'abstenir d'alcool» $[6,7]$.

\section{MODE D'ACTION DE L'ALCOOL}

L'alcool est constitué principalement d'éthanol $\left(\mathrm{CH}_{3} \mathrm{CH}_{2} \mathrm{OH}\right)$. Il est absorbé au niveau des muqueuses buccales, de l'estomac, du duodénum et de l'intestin grêle proximal par diffusion simple ne demandant aucune dépense énergétique. Il ne subit pas de stockage et doit être métabolisé et éliminé rapidement. Seulement $20 \%$ de l'alcool ingéré est métabolisé, le reste atteint la circulation sanguine pour être distribué en quelques minutes vers le foie, le cœur, les poumons, les reins et le cerveau (dont il imprègne largement les tissus). 5 à $10 \%$ de l'alcool ingéré sont éliminés dans l'urine, l'air expiré et la sueur, sans avoir subi une quelconque transformation, en effet l'essentiel de son métabolisme ( $90 \%)$ est pris en charge par le foie, qui l'élimine en le transformant d'abord en acétaldéhyde puis en acétate, grâce à l'intervention des enzymes de la famille des alcool-déshydrogénases (ADH), des aldéhydes déshydrogénases (ALDH) et du cytochrome CYP2E1. Enfin, l'acétate entre dans le cycle énergétique cellulaire, puis est oxydé en dioxyde de carbone et en eau dans les tissus. Lorsque l'alcoolémie est trop importante (plus de $0,3 \mathrm{~g} / \mathrm{L}$ dans le sang), le système $\mathrm{ADH}$ se sature et laisse place au système MEOS (Microsomal Ethanol Oxidizing System) dont le fonctionnement est autoentretenu par la consommation d'alcool elle-même. Ce système intervient également dans le métabolisme d'un grand nombre de médicaments, d'où la contreindication d'associer alcool et médicaments. L’organisme lélimine à raison de 0,10 à $0,15 \mathrm{~g} / \mathrm{L}$ par heure, soit une heure en moyenne par verre absorbé $[8,9,7]$.

\section{RISQUES À LONG TERME SUR LE PLAN GÉNÉRAL (en rapport avec notre spécialité)}

Les alcooliques chroniques présentent un risque infectieux élevé en raison de l'altération de leur système immunitaire. Ils souffrent d'une immunodéficience relative et sont donc vulnérables à toutes sortes d'infections, d'où la nécessité d'une antibioprophylaxie fréquente avant un acte sanglant. Ils sont également souvent porteurs d'affections hépatiques. En effet, 13 à $22 \%$ de la population alcoolique développent une cirrhose, les femmes étant plus touchées que les hommes. Cette pathologie est à lorigine de thrombopénie (diminution du nombre de plaquettes) et d'une baisse du taux de prothrombine (TP) exposant les patients à un risque hémorragique, d'autant plus qu'il existe une infection par le virus de l'hépatite B ou C $[7,10]$. Sachant que les valeurs normales se situent de 150000 à $40000010^{9}$ pour les plaquettes et à $100 \%$ pour le TP, dans le cas d'extractions dentaires, si le nombre de plaquettes est inférieur à $6000010^{9}$ par litre ou le TP à $60 \%$, il faut prévoir avec le service spécialisé, une transfusion de plaquettes en préopératoire. On comprend alors pourquoi les traitements de parodontologie et d'endodontie sont contre-indiqués chez ces patients. En effet, à cause des troubles généraux (immunodépression, carences), et des habitudes locales (hygiène déficiente, manque de maintenance), l'attitude du praticien peut être moins conservatrice et pencher vers l'avulsion devant le diagnostic de dents endodontiquement infectées ou de problèmes parodontaux importants [11].

Chez ces individus, l'ensemble des fonctions métaboliques est altéré. Parmi les expressions les plus courantes de l'usage chronique, on retrouve le diabète de type 2 [7]. La dénutrition suit de près, quelle soit primaire (baisse de la consommation d'aliments riches en micronutriments) [12-14] ou secondaire (lorsque l' absorption et le métabolisme d'un certain nombre de vitamines et de minéraux susceptibles de jouer un rôle protecteur dans la cancérogenèse sont altérés) $[15,16] .5$ à $30 \%$ des alcooliques chroniques souffrent de malnutrition [17], certains peuvent remplacer $60 \%$ de la ration calorique quotidienne par les calories fournies par l'alcool [18]. Ce sont certainement les sans domiciles fixes (SDF) qui en sont le plus atteints. Les déficiences nutritionnelles engendrées sont à l' origine de carences plus ou moins importantes : en protéines, vitamines (notamment en vitamine $\mathrm{K}$ ), minéraux (sélénium, zinc, magnésium), acide folique, riboflavine et interfèrent sur la santé tissulaire [16].

\section{ALCOOL ET CAVITÉ BuCCALE}

Les effets de l'alcool sur le milieu buccal sont nombreux. Une des premières constatations du professionnel de santé est l'halitose. En effet, il semblerait qu'il existe une relation entre consommation d'alcool (en particulier consommation quotidienne) et mauvaise haleine. Cette dernière est induite par des composés sulfurés volatils (CSV), les mêmes que l'on retrouve dans les œufs pourris, produits par les bactéries gram négatif de la cavité buccale. On sait que certains facteurs peuvent provoquer la prolifération de ces bactéries comme la xérostomie, l'existence de cavités carieuses, les nécroses pulpaires à ciel ouvert et les parodontites. Cependant, halitose, alcool et maladies parodontales avec poches supérieures ou égales à 5 millimètres seraient communément associés [19, 20]. En effet, l'alcool joue un rôle sur la salive et modifie le biofilm [8]. Dès son absorption, l'alcool (éthanol) diffuse rapidement dans la salive, à tel point que sa concentration salivaire est supérieure à celle du plasma (pendant les 30 premières minutes) [21]. Ceci suggère que l'éthanol pénètre facilement l'ensemble du corps incluant les tissus buccaux et les glandes salivaires. 
Au niveau oral, il altère la morphologie et la fonction des ces dernières, diminuant ainsi le débit salivaire. En effet, il provoque une sialadénite, à l'origine d'une hyposialie qui favorise la croissance des bactéries cariogènes [22]. Or la salive joue un rôle important dans l'élimination mécanique des micro-organismes et des débris alimentaires, mais aussi en maintenant le $\mathrm{pH}$ (rôle tampon) et en détruisant les bactéries par l'action des immunoglobulines et des enzymes [23]. Ce phénomène est aggravé par la mauvaise hygiène locale souvent associée à l'abus d'alcool. Au niveau général, l'acétaldéhyde issu du métabolisme de l'éthanol par le foie est considéré comme un produit toxique et cancérigène qui expliquerait l'élévation du risque de cancers non spécifiques chez les alcooliques [24]. Au niveau local, l'exposition directe à ce produit (présent dans certains alcools forts comme le cognac et le calvados) augmente sa concentration salivaire (pendant au moins 10 minutes après l'ingestion), indépendamment de sa distribution systémique qui a lieu à partir de la circulation sanguine $[25,26]$. Ces taux salivaires élevés d'acétaldéhyde sont également induits par sa production locale par certaines bactéries salivaires qui métabolisent l'éthanol en acétaldéhyde directement au contact de la muqueuse buccale grâce à leur enzyme alcool déshydrogénase [27-29]. Le phénomène est aggravé par la baisse du débit salivaire responsable d'une hausse de la concentration bactérienne, et par la mauvaise hygiène buccale souvent de règle chez les buveurs $[30,8]$.

Les tissus durs sont donc plus exposés à l'action des bactéries et les tissus mous aux microbes et aux agents cancérigènes dont l'acétaldéhyde, avec comme conséquence un risque accru de lésions muqueuses incluant dysplasies, leucoplasies ou carcinomes [8].

\section{EFFETS DE L'ALCOOL SUR LES TISSUS DURS}

\section{Sur la dent}

Il existe une association positive entre consommation d'alcool et plus grande incidence de caries et de pertes dentaires [31] (fig. 2), même si pour certains, cette corrélation serait causée en grande partie par un manque

\footnotetext{
Fig. 3 a :

Lésions érosives au niveau des bords

libres des incisives mandibulaires et maxillaires.
}

d'hygiène et des conditions socio-économiques défavorables [32]. Cependant, un régime alimentaire associé à des boissons à base de saccharose et d'alcool a tendance à augmenter la colonisation par Streptococcus mutans et l'incidence des caries sur les faces occlusales des molaires et les surfaces lisses des autres dents, par rapport à un régime de contrôle. Le saccharose a été identifié comme étant le substrat fondamental pour la synthèse de glucane extracellulaire (polysaccharide composé exclusivement de monomère de glucose), qui est responsable de la capacité des Streptococcus mutans à adhérer à la surface lisse des dents [33]. Toutes les boissons alcoolisées sont sucrées et acides. En cas de consommation journalière régulière, le $\mathrm{pH}$ buccal est sans cesse abaissé, ce qui, associé à l'hyposialie de règle dans ce cas, a un impact direct sur la formation de carie.

Une autre manifestation possible de la prise d'alcool en excès est l'érosion dentaire qui se manifeste par une perte plus ou moins importante des tissus durs de la dent. Cette dernière peut s'expliquer par les régurgitations et les affections gastriques dues à l'absorption d'une quantité importante chronique de ce breuvage au $\mathrm{pH}$ acide (environ 3). On sait qu'il existe d'ailleurs une diminution significative du $\mathrm{pH}$ salivaire chez les patients gros consommateurs d'alcool $[34,35]$. Les faces palatines des dents antérieures suivies respectivement des bords incisifs et des faces occlusales des dents postérieures seraient les plus affectés par ces lésions érosives à morphologie spécifique (aspect en cuvette, à fond régulier et bord nets) [36, 23] (fig. 3). Certaines études in vitro soulignent que les vins blancs auraient un pouvoir plus abrasif que les vins rouges [37]. À ce sujet d'ailleurs, des colorations peuvent être constatées car, à l'instar du tabac et du café, l'alcool, en particulier le vin rouge (en raison de la présence de tanins) peut provoquer des dyschromies, dues à la porosité de l'émail facilement pénétré par les colorants alimentaires. Sa consommation doit donc être

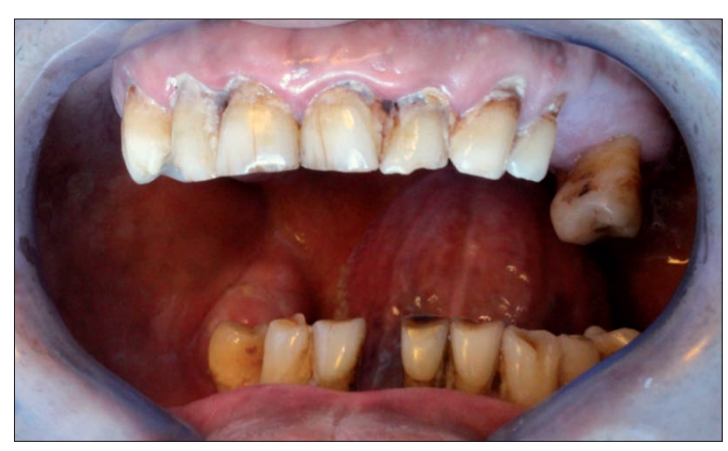

4 Fig. 2 :

Mauvais état dentaire chez un alcoolique.

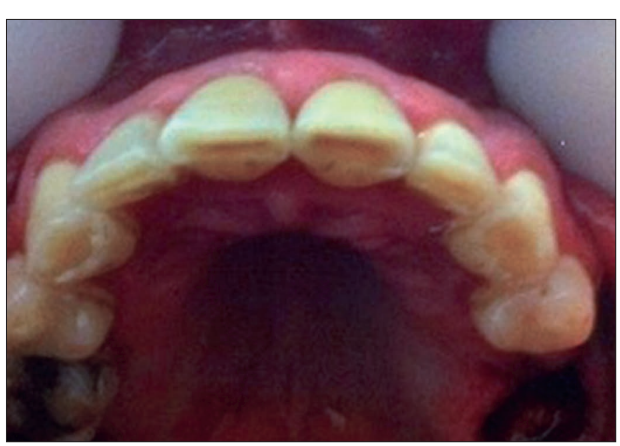

$\triangle$ Fig. 3 b :

Lésions érosives au niveau des bords libres et des faces occlusales au maxillaire. 
vivement déconseillée après un "blanchiment ", la porosité de l'émail étant accentuée dans ce cas [38].

\section{Sur l'os}

Sur le plan général, l'abus d'alcool a un rôle néfaste sur la santé osseuse et représente un facteur de risque d'ostéoporose, de fractures et de non-consolidation après fractures [39-42]. Bien que les mécanismes par lesquels ses effets sur le métabolisme osseux et la balance entre résorption et apposition autour d'un implant (quel qu'il soit) ne soient pas complètement élucidés [43], on sait que sa consommation est associée à des déficiences du système immunitaire qui perturbent le remaniement des tissus osseux péri-implantaires, mettant en péril l'ostéo-intégration $[44,45]$ et favorisant la péri-implantite [46].

\section{EFFETS DE L'ALCOOL SUR LES TISSUS MOUS}

Les tissus durs ne sont pas les seuls affectés par la consommation d'alcool, les répercussions sur les tissus gingivaux et muqueux sont légion : en effet à cause des déficiences nutritionnelles et des carences induites, du reflux gastro-œsophagien, de facteurs psychologiques assez constants (stress, angoisse, dépression), le développement et la progression de la maladie parodontale sont exacerbés. De récentes études mettent l'accent sur l'association stress-maladies parodontales via des mécanismes physiologiques et comportementaux [47, 48]. Au niveau général, le stress et l'état dépressif diminuent la réponse immunitaire et favorisent l'inflammation chronique. Ces effets seraient dépendants de l'augmentation du taux de cortisol salivaire (hormone glucocorticoïde) qui diminue l'immunocompétence en inhibant les immunoglobulines $\mathrm{A}$ et $\mathrm{G}$ et la fonction des polynucléaires neutrophiles [48]. Au niveau comportemental, des habitudes néfastes comme le tabagisme, une médiocre hygiène alimentaire et orale, fréquemment associées à l'abus d'alcool exacerbent la réponse à l'agression.

Dans le contexte d'altérations de létat général, de déficiences nutritionnelles et de carences, on retrouve des retards de cicatrisation, des candidoses, des glossites, des blessures sous-prothétiques et des lésions muqueuses [23].
Les retards de cicatrisation peuvent avoir lieu après une simple extraction. Sous l'effet de l'alcool, l'accumulation de protéines et de collagène se fait mal dans l'alvéole déshabitée, l'activation et la prolifération des lymphocytes T sont diminuées ainsi que la capacité de mobilisation et de phagocytose des macrophages et des neutrophiles. Les candidoses, elles, vont pouvoir s'installer grâce au déséquilibre de la flore commensale, à l'acidification du milieu buccal et à la diminution de la réponse immunitaire [49]. Chez les porteurs de prothèses amovibles, elles sont préférentiellement sous-prothétiques, l'intrados représentant un véritable réservoir à Candidas [50] (fig. 4). D'ailleurs, l'inconfort voire l'intolérance au port prothétique est fréquent(e), à cause de la santé tissulaire défaillante, de la xérostomie, de l'hygiène (orale et prothétique) aléatoire, d'une éventuelle candidose, d'autant plus que le matériel prothétique a subi un vieillissement prématuré par action de l'éthanol sur les propriétés physiques de la résine acrylique [51].

S'agissant d'anomalies muqueuses, bien que controversé en tant qu'étiologie à part entière, car souvent combiné au tabac, l'alcool ferait le lit des lésions précancéreuses [52-55] telles que les leucoplasies homogènes ou inhomogènes $[56,57]$. La leucoplasie est une lésion blanche kératosique de la muqueuse buccale qui ne peut être détachée par le grattage. Elle est asymptomatique. Toutes les parties orales peuvent être touchées avec une prédominance pour la commissure labiale, les joues et le bord de la langue. On distingue la leucoplasie homogène (fig. 5) qui se présente sous forme de plaque blanche nettement circonscrite, peu ou pas surélevée, parfois légèrement fissurée et craquelée, sans inflammation périlésionnelle et sans adénopathie satellite. Elle est réversible et sa transformation maligne est assez rare (5\% des cas). Les leucoplasies inhomogènes, elles, comprennent les formes nodulaires, exophytiques et les érythroleucoplasies (fig. 6). Elles se manifestent soit sous forme d'un nodule blanchâtre, soit d'une plage verruqueuse blanche, nacrée, parfois fissurée, soit d'une lésion combinant un aspect érythémateux à un aspect blanchâtre au relief et aux bords très irréguliers. Les caractères cliniques particuliers en font des lésions au risque élevé de cancérisation. Quoi qu'il en soit, il est certain que l'abus d'alcool potentialise l'action du tabac et, s'il n'intervient pas directement dans la kératose, il intervient sûrement dans les facteurs de cancérisation [58-61].

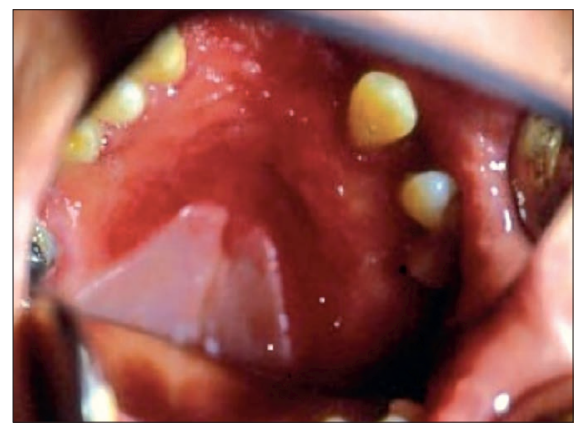

$\triangle$ Fig. 4 :

Candidose palatine sous-prothétique.

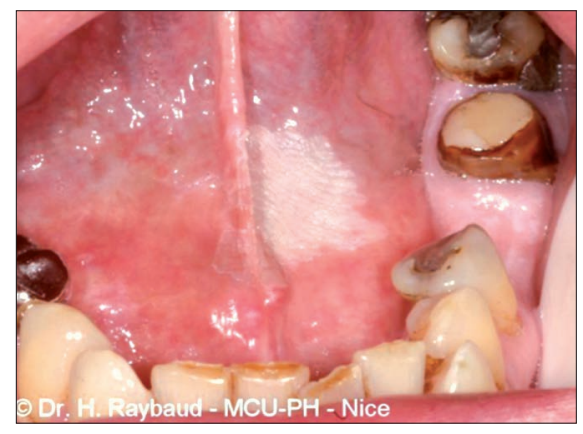

$\triangle$ Fig. 5 :

Leucoplasie homogène du plancher lingual.

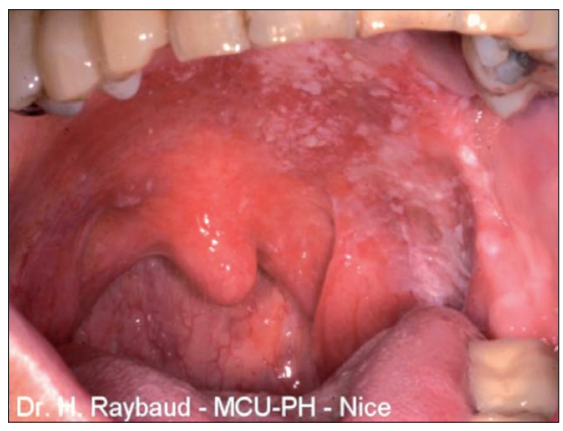

Fig. 6 :

Leucoplasie inhomogène : érythroleucoplasie. 
En effet, l'alcool altère la muqueuse buccale par action directe sur la bicouche phospholipidique qui constitue la membrane des cellules muqueuses. Le principe est simple : en cas d'exposition à un solvant tel que léthanol qui élimine certains des lipides constitutifs de la membrane cellulaire, celle-ci devient alors beaucoup plus perméable, d'où une pénétration accrue d'agents cancérogènes et une sensibilité à ceux-ci plus importante car le produit altère les mécanismes de réparation de l'ADN [8, 62]. On sait que de nombreuses tumeurs malignes sont imputables à l'alcool, notamment les cancers des voies aérodigestives supérieures (VADS) qui regroupent les cancers de la cavité buccale (lèvre-bouche-pharynx) associés aux cancers du larynx et des sinus de la face [9]. En France, ils représentent $10 \%$ de l'ensemble des cancers [63]. Malgré une baisse de leur incidence dans notre pays depuis le début des années 1980, le nombre de nouveaux cas reste important comparé au reste de l'Europe, plaçant la France au premier rang notamment pour les cancers de la cavité orale et du larynx. En effet plus de 6000 nouveaux cas de cancers de la cavité buccale sont diagnostiqués chaque année, ils représentent $3 \%$ de l'ensemble des tumeurs malignes et $30 \%$ des cancers des VADS [64].

Ces tumeurs surviennent 9 fois sur 10 sur un terrain alcoolo-tabagique, le carcinome épidermoïde de la muqueuse buccale représentant $95 \%$ des cancers de la cavité orale. Ces derniers sont les seuls qui n'aient pas connu d'augmentation du taux de survie depuis 30 ans [65].

Le carcinome épidermoïde (fig. 7) peut être retrouvé sur toutes les muqueuses orales mais il se développe le plus souvent au niveau de la langue $(25 \%)$, du plancher buccal (17\%) et des gencives (13\%). Il se décline sous plusieurs formes cliniques (ulcéreuse, végétante, ulcérovégétante et nodulaire interstitielle) [59]. Peu importe la localisation topographique et la forme clinique, ces carcinomes présentent une caractéristique commune : existence d'une induration à la palpation s'étendant bien au-delà de la tumeur. En général, ils restent longtemps asymptomatiques, indolores, du moins pour les formes peu évoluées survenant sur une muqueuse saine. En revanche, s'ils se développent à partir d'une lésion préexistante, ils peuvent s'accompagner de douleurs. Parmi les symptômes les plus souvent rencontrés, on retrouve des sensations de picotements, de brûlures et une halitose toujours très prononcée.

Fig. 7 :

Carcinome épidermoïde de la gencive.

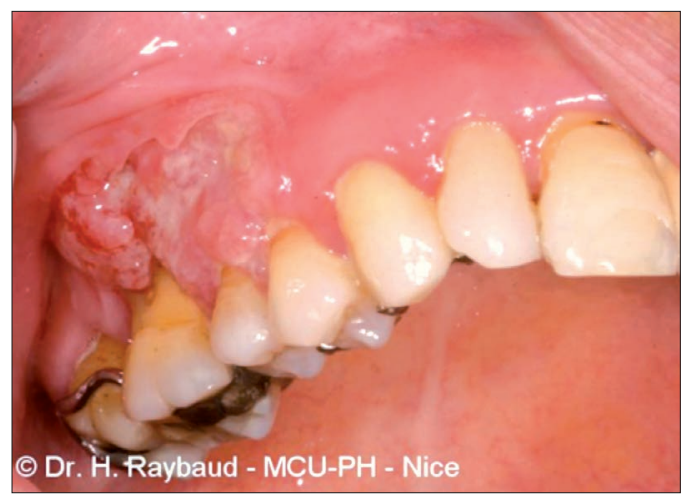

\section{RELATION DOSE-EFFET ET MODALITÉS DE CONSOMMATION}

Le lien positif entre l'apparition d'un cancer des VADS et la consommation régulière d'alcool, indépendamment de la consommation de tabac, est établi depuis de nombreuses années [9]. Cette corrélation est fonction de la quantité d'alcool absorbée en moyenne par jour et de l'ancienneté de la consommation [66-68].

Par rapport aux non-buveurs, le risque est multiplié par deux chez les individus qui consomment 10 grammes par jour (soit 1 verre), il est décuplé pour les consommateurs de 50 à $60 \mathrm{~g}$ par jour (5 à 6 verres) et il est multiplié par 13,7 pour les consommateurs dépassant $110 \mathrm{~g} / \mathrm{j}[69,70]$. L'importance du type d'alcool (vin, bière, spiritueux) a été étudiée pour la première fois en 1988 par un groupe d'experts internationaux (IARC, 1988), puis réévaluée par la suite. La majorité des auteurs s'accordent pour dire que la durée et la quantité d'alcool consommée semblent être des facteurs de risque plus importants que le type de boissons alcoolisées absorbées [69-74]. S’agissant du cancer buccal, le risque diminue au bout de trois ans d'arrêt, demandant au moins quatorze années pour être proche de celui de la population abstinente et une vingtaine d'années pour le cancer du larynx $[69,70]$.

\section{INTERACTION AVEC D'AUTRES FACTEURS DE RISQUE : LE TABAC}

Quarante-deux pour cent des sujets qui consomment plus de 3 verres d'alcool par jour déclarent fumer [65]. Si, pour les consommateurs d'alcool, le risque de développer un cancer des VADS est démontré, le risque devient nettement plus important lorsque le sujet associe alcool et tabac. En effet, ces deux facteurs agissent en synergie, ils ne s'additionnent pas, ils se potentialisent [75-79]. Le risque peut être multiplié par 45 pour un même fumeur selon la quantité d'alcool qu'il consomme [9]. En fait, près de $90 \%$ des cancers des VADS pourraient être évités si la population ne fumait pas et buvait moins d'un demi-litre de vin par jour (soit 4 verres par jour) [63].

\section{CONSÉQUENCES TRAUMATIQUES}

Le coût total de la consommation d'alcool pour la société est compris entre 1 et $3 \%$ du produit intérieur brut en comprenant le coût des pertes de productivité, celui des accidents, les dépenses de santé et les coûts pour la collectivité liés aux crimes et délits [80, 81]. En effet, les risques encourus peuvent être majeurs : diminution de la vigilance, souvent responsable d'accidents de la circulation et d'accidents du travail, perte de 
contrôle de soi conduisant à des comportements violents, à des passages à l'acte, agressions sexuelles, suicide, homicide, enfin exposition à des agressions en raison d'une attitude provocatrice ou du fait que l'état d'ébriété puisse empêcher de se défendre $[1,82]$. En fait, l'alcool pousse l'individu à commettre des actes lui portant préjudice [83], l'un des impacts de cette drogue licite sur la cavité buccale est traumatique, canettes décapsulées avec les dents, rixes, chutes, accidents [10]. Aux urgences dentaires, l'accueil des traumas est quotidien et peut aller de la simple contusion aux luxations et fractures dentaires et maxillaires incluant plaies et dilacérations des lèvres et des gencives (fig. 8, 9 et 10).

\section{RÔLE DU CHIRURGIEN-DENTISTE}

Le patient dépendant à l'alcool n'est pas un patient comme les autres. Sa prise en charge nécessite une connaissance précise du produit consommé, de ses effets généraux et de ses conséquences sur la cavité buccale. Si les mesures politiques n'arrivent toujours pas à inciter les buveurs excessifs à réduire ou à stopper leur consommation, le discours médical peut parfois être l'élément déclencheur à la prise de conscience de la réalité de l'addiction. L'accompagnement du malade alcoolique s'inscrit dans le temps et nécessite un travail d'équipe simultané et concerté car l'alcoolisme est une pathologie globale, médicale, sociale et psychologique. Le but est d'inciter le patient à se prendre en charge. À la moindre suspicion de toxicomanie alcoolique, le praticien doit pouvoir évoquer le sujet, en parler sans tabou, sans jugement, sans diabolisation, afin de transmettre au patient des informations claires, le mettre en garde contre les risques encourus et l'orienter vers des centres spécialisés (centre d'addictologie hospitalier, médecin addictologue, centre associatif d'accueil et d'écoute type CAARUD (Centres d'accueil et d'accompagnement à la réduction des risques pour usagers de drogues) ou CSAPA (Centres de soins d'accompagnement et de prévention en addictologie). N'oublions pas que le chirurgien-dentiste peut avoir un rôle gâchette car la réhabilitation orale fait partie intégrante des protocoles de sevrage et de réinsertion sociale (esthétique et nutrition). D'autre part, les praticiens sont bien placés pour faire passer

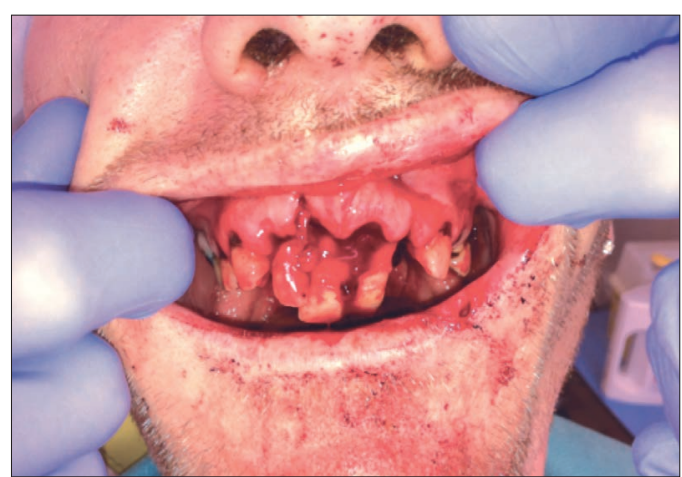

$\triangleleft$ Fig. 8:

Fracture alvéolaire maxillaire antérieure et extrusion complète de 12,11 , et 21 (la 22 n'a pas été retrouvée), suite à une rixe.

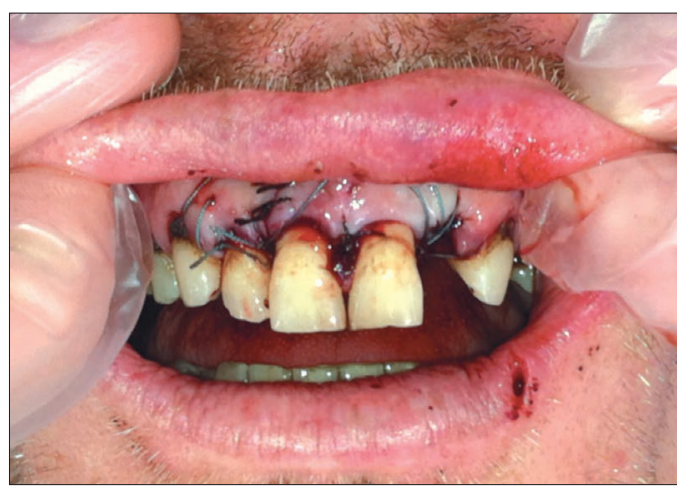

$\triangle$ Fig. 9 :

Remise en place des dents dans leurs alvéoles et suture de la gencive.

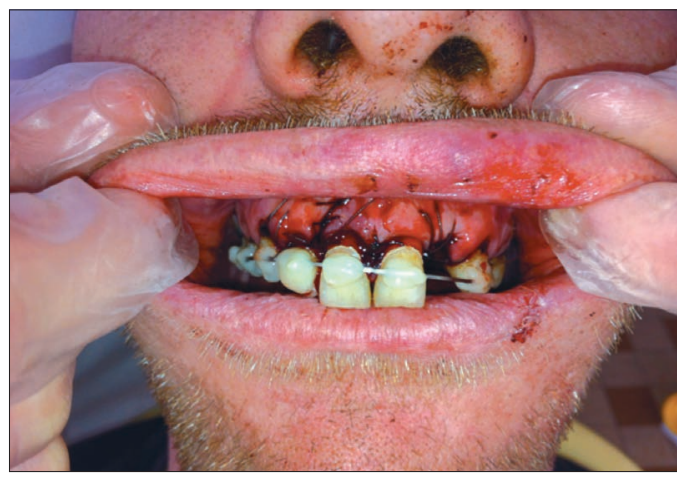

Fig. 10 :

Attelle de contention en place.

des messages de prévention auprès des jeunes, sur les effets délétères de cette substance addictive qui accélère le vieillissement et a une action néfaste sur les dents, le parodonte et les muqueuses. Ils jouent également un rôle fondamental dans la prévention et le dépistage d'éventuelles lésions précancéreuses et cancéreuses. En effet, le suivi régulier et le dépistage précoce restent les moyens les plus efficaces de faire baisser la morbidité et la mortalité des cancers de la cavité buccale.

\section{Bibliographie}

[1] Institut national de prévention et d'éducation pour la santé (Inpes). Drogues et dépendances. Le livret d'information. SaintDenis : Editions INPES, 2007.
[2] Choi NG, Dinitto DM. Heavy/binge drinking and depressive symptoms in older adults: gender differences. Int J Geriatr Psychiatry 2011;26(8):860-8.

[3] Cadet-Tairou A, Gandilhon M, Toufik A, Evrard I. Phénomènes émergents liés aux drogues en 2006. Huitième rapport national du dispositif TREND. Saint-Denis : Edition OFDT, 2008.

[4] Com-Ruelle L, Dourgnon P, Jusot F, Lengagne P. Les problèmes d'alcool en France : quelles sont les populations à risque ? IRDES, Questions d'économie de la santé 2008;129:1-6. 
[5] Veissière P. Kit de secours pour alcoolique. Allainville-aux-bois : Editions Grrr... Art, 2011.

[6] Institut national de prévention et d'éducation pour la santé (Inpes). Drogues et dépendances. Le livret d'information. Saint-Denis : Editions INPES, 2007.

[7] Richard D, Senon JL, Valleur M. Dictionnaire des drogues et des dépendances. Lamnay : Editions Larousse, 2009.

[8] Riedel F, Goessler UR, Hormann K. Alcohol-related diseases of the mouth and throat. Dig Dis 2005;23(3-4):195-203.

[9] Institut national du cancer. Alcool et risques de cancers. Etats des lieux des données scientifiques et recommandations de santé publique. Boulogne Billancourt : Editions INCa, Collection Rapports et synthèse, 2007.

[10] Pesci-Bardon C, Prêcheur I. Conduites addictives : tabac, alcool, psychotropes et drogues illicites. Impact sur la santé buccodentaire. EMC (Elsevier Masson SAS, Paris), Médecine buccale, 28-915-M-10, 2010, Odontologie, 23-760-A-40, 2011.

[11] Khocht A, Janal M, Schleifer S, Keller S. The influence of gingival margin recession on loss of clinical attachment in alcoholdependent patients without medical disorders. J Periodontol 2003;74(4):485-93.

[12] Seitz HK, Pöschl G, Simanowski UA. Alcohol and cancer. Recent Dev Alcohol 1998;14:67-95.

[13] Lieber CS. Hepatic, metabolic, and nutritional disorders of alcoholism: from pathogenesis to therapy. Crit Rev Clin Lab Sci 2000;37(6):551-84.

[14] Lieber CS. Relationships between nutrition, alcohol use, and liver disease. Alcohol Res Health 2003;27(3):220-31.

[15] Maillot F, Farad S, Lamisse F. Alcohol and nutrition. Pathol Biol 2001;49(9):683-8.

[16] Boffetta P, Hashibe M. Alcohol and cancer. Lancet Oncol 2006;7(2):149-56.

[17] Piquet MA. Alcoolisme : prise en charge nutritionnelle. Cah Nutr Diét 2002;37(2):136-40.

[18] Bunout D. Nutritional and metabolic effects of alcoholism : their relationship with alcoholic liver disease. Nutrition 1999;15(7-8):583-89.

[19] van den Velde S, Quirynen M, van Hee P, van Steenberghe D. Halitosis associated volatles in breath of healthy subjects. J Chromatogr $B$ Analyt Technol Biomed Life Sci 2007;853(1-2):54-61.

[20] Suzuki N, Yoneda M, Naito T, Iwamoto T, Yamada K, Hisama K, Okada I, Hirofuji T. The relationship between alcohol consumption and oral malodour. Int Dent J 2009;59(1):31-4.
[21] Waszkiewicz N, Zalewska A, Szulc A, Kepka A, Konarzewska B, Zalewska-Szajda B, Chojnowska S, Waszkiel D, Zwierz K. The influence of alcohol on the oral cavity, salivary glands and saliva. Pol Merkur Lekarski 2011;30(175):69-74.

[22] Prestifilippo JP, Fernandez-Solari J, Medina V, Rettori V, Elverdin JC. Role of the endocannabinoid system in ethanolinduced inhibition of salivary secretion. Alcohol Alcohol 2009;44(5):443-8.

[23] Mainguet P. Les manifestations supraglottiques du RGO. Nancy : Editions ALN, 2003.

[24] Balbo S, Meng L, Bliss RL, Jensen JA, Hatsukami DK, Hecht SS. Kinetics of DNA adduct formation in the oral cavity after drinking alcohol. Cancer Epidemiol Biomarkers Prev 2012;21(4):601-8.

[25] Lachenmeier DW, Monakhova YB. Short-term salivary acetaldehyde increase due to direct exposure to alcoholic beverages as an additional cancer risk factor beyond ethanol metabolism. J Exp Clin Cancer Res 2011 janv 6;30(1):3.

[26] Lindenborg K, Salaspuro M, Väkeväinen S. A single sip of a strong alcoholic beverage causes exposure to carcinogenic concentrations of acetaldehyde in the oral cavity. Food Chem Toxicol 2011;49(9):2103-6.

[27] Kurkivuori J, Salaspuro V, Kaihovaara P, Kari K, Rautemaa R, Grönroos L, Meurman JH, Salaspuro M. Acetaldehyde production from ethanol by oral streptococci. Oral Oncol 2007;43(2):181-6.

[28] Meurman JH, Uittamo J. Oral micro-organisms in the etiology of cancer. Acta Odontol Scand 2008;66(6):321-6.

[29] Yokoyama A, Tsutsumi E, Imazeki H, Suwa Y Nakamura C, Mizukami T, Yokoyama T. Salivary acetaldehyde concentration according to alcoholic beverage consumed and aldehyde dehydrogenase- 2 genotype. Alcohol Clin Exp Res 2008;32(9):1607-14.

[30] Homann N, Tillonen J, Rintamäki H, Salaspuro M, Lindqvist C, Meurman JH. Poor dental status increases acetaldehyde production from ethanol in saliva: a possible link to increased oral cancer risk among heavy drinkers. Oral Onco 2001;37(2):153-8.

[31] Rooban T, Vidya K, Joshua E, Rao A, Ranganathan S, Rao UK, Ranganathan K. Tooth decay in alcohol and tobacco abusers. J Oral Maxillofac Pathol 2011;15(1):14-21.

[32] Dasanayake AP, Warnakulasuriya S, Harris CK, Cooper DJ, Peters TJ, Gelbier S. Tooth decay in alcohol abusers compared to alcohol and drug abusers. Int J Dent 2010;2010:786503. Epub 2010 Apr 1. Published on line.
[33] Kantorski KZ, de Souza DM, Yujra VQ, Junqueira JC, Jorge AO, da Rocha RF. Effect of an alcoholic diet on dental caries and on Streptococcus of the mutans group. Study in rats. Braz Oral Res 2007;21(2):101-5.

[34] Chehal HK, Pate DH, Cohen DM, Battacharyya I. Dental erosion due to excessive wine consumption. Gen Dent 2009;57(5):519-23.

[35] Dukić W, Dobrijević TT, Katunarić M, Milardović S, Segović S. Erosive lesions in patients with alcoholism. J Am Dent Assoc 2010;141(12):1452-8.

[36] Manarte P, Manso MC, Souza D, Frias-Bulhosa J, Gago S. Dental erosion in alcoholic patients under addiction rehabilitation therapy. Med Oral Patol Oral Cir Bucal 2009;14(8):e376-83.

[37] Willershausen B, Callaway A, Azrak B, Kloss C, Schulz-Dobrick B. Prolonged in vitro exposure to white wines enhances the erosive damage on human permanent teeth compared with red wines. Nutr Res 2009;29(8):558-67.

[38] Liporoni PC, Souto CM, Pazinatto RB, Cesar IC, de Rego MA, Mathias P, Cavalli V. Enamel susceptibility to coffee and red wine staining at different intervals elapsed from bleaching: a photoreflectance spectrophotometry analysis. Photomed Laser Surg 2010;28 Suppl 2:S105-9.

[39] Black DM, Cooper C. Epidemiology of fractures and assessment of fracture risk. Clin Lab Med 2000;20(3):439-53.

[40] Volkmer DL, Sears B, Lauing KL, Nauer RK, Roper PM, Yong S, Stover M, Callaci JJ. Antioxidant therapy attenuates deficient bone fracture repair associated with binge alcohol exposure. J Orthop Trauma 2011;25(8):516-21.

[41] Drake MT, Murad MH, Mauck KF, Lane MA, Undavalli C, Elraiyah T, Stuart LM, Prasad C, Shahrour A, Mullan RJ, Hazem A, Erwin PJ, Montori VM. Clinical review. Risk factors for low bone mass-related fractures in men: a systematic review and meta-analysis. J Clin Endocrinol Metab 2012;97(6):1861-70.

[42] Legrand E, Hoppé E, Bouvard B, Audran M. Osteoporosis in men. Rev Prat 2012;62(2):193-7.

[43] Fini M, Giavaresi G, Salamanna F, Veronesi F, Martini L, De Mattei M, Tschon M. Harmful lifestyles on orthopaedic implantation surgery: a descriptive review on alcohol and tobacco use. J Bone Miner Metab 2011;29(6):633-44.

[44] Camilli JA, da Cunha MR, Bertran CA, Kawachi EY. Subperiosteal hydroxyapatite implants in rats submitted to ethanol ingestion. Arch Oral Biol 2004;49(9):747-53.

[45] Alissa R, Olivier RJ. Influence of prognostic 
risk indicators on osseointegrated dental implant failure: a matched case-control analysis. J Oral Implantol 2012;38(1):51-61.

[46] Nguyen-Hieu T, Borghetti A, Aboudharam G. Peri-implantitis: from diagnosis to therapeutics. J Investig Clin Dent 2012;3(2):79-94.

[47] Goyal S, Jajoo S, Nagappa G, Rao G. Estimation of relationship between psychosocial stress and periodontal status using serum cortical level: a clinicobiochemical study. Indian J Dent Res 2011;22(1):6-9.

[48] Rai B, Kaur J, Anand SC, Jacobs R. Salivary stress markers, stress, and periodontitis: a pilot study. J Periodontol 2011;82(2):287-92.

[49] Gauzeran D. Candidoses buccales. Paris : Éd. AC3P-Pred, 2000.

[50] Pesci-Bardon C, Fosse T, Serre D, Madinier I. In vitro antiseptic properties of an ammonium compound combined with denture base acrylic resin. Gerodontology 2006;23(2):111-6

[51] Regis RR, Soriani NC, Azevedo AM, Silva-Lovato CH, Paranhos HF, de Souza RF. Effects of ethanol on the surface and bulk properties of a microwave-processed PMMA denture base resin. J Prosthodont 2009;18(6):489-95.

[52] Maserejian NN, Joshipura KJ, Rosner BA, Giovannucci E, Zavras AI. Prospective study of alcohol consumption and risk of oral premalignant lesions in men. Cancer Epidemiol Biomarkers Prev 2006;15(4):774-81.

[53] Saraswathi TR, Ranganathan K, Shanmugam S, Sowmya R, Narasimhan PD, Gunaseelan R. Prevalence of oral lesions in relation to habits: Cross-sectional study in South India. Indian J Dent Res 2006;17(3):121-5

[54] Burzlaff JB, Bohrer PL, Paiva RL, Visioli F, Sant'Anna Filho M, da Silva VD, Rados PV. Exposure to alcohol or tobacco affects the pattern of maturation in oral mucosal cells: a cytohistological study. Cytopathology 2007;18(6):367-75.

[55] Riebel J, Holmstrup P. Premalignant disorders and cancer of the oral mucosa. Ugeskr Laeger 2010;172(44):3040-2.

[56] Zhang X, Li C, Song Y, Reichart PA. Oral leukoplakia in China: a review. Oral Maxillofac Surg 2010;14(4):195-202.

[57] Pigolkin lul, Dolzhanskii OV, Mamsurova TS, Chertovskikh AA. Forensic medical diagnostics of chronic alcoholic intoxication based on histological changes in the soft tissues of oral cavity and salivary glands. Sud Med Ekspert 2011;54(3):10-2.

[58] Ben Slama L. Lésions précancéreuses de la muqueuse buccale. Rev Stomatol Chir Maxillofac 2001;102(2):77-108.

[59] Gauzeran D. Dépistage des cancers de la cavité orale. Les Cahiers de l'ADF 2003;16:10-6.

[60] Gauzeran D. Lesions à risque et cancers de la bouche : diagnostic et traitement. RueilMalmaison : Éd. CDP, Coll. Mémento, 2007.

[61] Piette E, Reychler H. Lésions blanches de la muqueuse et des lèvres. EMC (Elsevier Masson SAS, Paris), Médecine buccale, Stomatologie,22-045-K-10, 2006, Médecine buccale, 28-280-G-10, 2008.

[62] Sergent O, Griffon B, Cillard P., Cillard J. Alcohol and oxidative stress. Pathol Biol 2001;49(9):689-95.

[63] Brugère J, Schwaab G. Le cancer des voies aérodigestives supérieures : information et prévention. Paris : Editions Ligue nationale contre le cancer, 2002 (mise à jour 2006).

[64] Féki A, Abi Najm S, Descroix V, Gauzeran D, Gourmet R, Guichard M, Kleifinger S, Lescaille G, Lombardi T, Samson J. Le chirurgien-dentiste face au cancer. Du diagnostic précoce du cancer buccal à la prise en charge du patient cancéreux. Les dossiers de l'ADF. Paris : Edition ADF, 2008.

[65] L'essentiel sur les cancers de la cavité buccale. Dossier de presse. Paris : Edition INC, 2011.

[66] Grønbaek M, Becker U, Johansen D, Tønnesen H, Jensen G, Sørensen TI. Population based cohort study of association between alcohol intake and cancer of the upper digestive tract. BMJ 1998;317(7162):844-7.

[67] Bagnardi V, Blangiardo M, La Vecchia C Corrao G. A meta-analysis of alcohol drinking and cancer risk. Br J Cancer 2001;85(11):1700-05

[68] Zeka A, Gore R, Kriebel D. Effects of alcohol and tabaco on aerodigestive cancer risks: a meta regression analysis. Cancer Causes Control 2003;14(9):897-906.

[69] Altieri A, Bosetti C, Gallus S, Franceschi S, Dal Maso L, Talamini R, Levi F, Negri E, Rodriguez T, La Vecchia C. Wine, beer and spirits and risk of oral and pharyngeal cancer: a case of control study from Italy and Switzerland. Oral Oncol 2004;40(9):904-9.

[70] Cactellsagué X, Quintana MJ, Martinez MC, Nieto A, Sanchez MJ, Juan A, Monner A, Carrera M, Agudo A, Quer M, Muñoz N, Herrero R, Franceschi S, Bosch FX. The role of type of tabaco and type of alcoholic beverage in oral carcinogenesis. Int $J$ Cancer 2004;108(5):741-9.

[71] Kabat GC, Wynder EL. Type of alcoholic beverage and oral cancer. Int J Cancer 1989;43(2):190-4.
[72] Barra S, Franceschi S, Negri E, Talamini R, La Vecchia C. Type of alcoholic beverage and cancer of the oral cavity, pharynx and oesophagus in an Italian area with high wine consumption. Int J Cancer 1990;46(6):1017-20

[73] Grønbaek M, Becker U, Johansen D, Tønnesen H, Jensen G, Sørensen TI. Population based cohort study of association between alcohol intake and cancer of the upper digestive tract. $B M J$ 1998;317(7162):844-7

[74] Bosetti C, La Vecchia C, Negri E, Franceschi S. Wine and other types of alcoholic beverages and the risk of esophageal cancer. Eur J Clin Nutr 2000;54(12):918-20.

[75] Blot WJ, McLaughlin JK, Winn DM, Austin DF, Greenberg RS, Preston-Martin S, Bernstein L, Schoenberg JB, Stemhagen A, Fraumeni JF Jr. Smoking and drinking in relation to oral and pharyngeal cancer. Cancer Res 1988;48(11):3282-7.

[76] Choi SY, Kahyo H. Effect of cigarette smoking and alcohol consumption in the aetiology of cancer of the oral cavity, pharynx and larynx. Int J Epidemiol 1991;20(4):878-85.

[77] Hayes RB, Bravo-Otero E, Kleinman DV, Brown LM, Fraumenti JF Jr, Harty LC, Winn DM. Tobacco and alcohol use and oral cancer in Puerto Rico. Cancer Causes Control 1999;10(1):27-33.

[78] Castellsagué X, Muñoz N, De Stefani E, Victora CG, Castelletto R, Rolón PA, Quintana MJ. Independent and joint effects of tobacco smoking and alcohol drinking on the risk of esophageal cancer in men and women. Int J Cancer 1999;82(5):657-64.

[79] Taylor B, Rehm J. When risk factors combine: the interaction between alcohol and smoking for aerodigestive cancer, coronary heart disease, and traffic and fire injury. Addict Behav 2006;31(9):1522-35.

[80] Leifman H, Österberg E, Ramstedt M. European comparative alcohol study (ECAS). Stockholm: Edita Ljunglöfs i stockholm, 2002.

[81] Anderson P, Baumberg B. Alcohol in Europe. London: Institute of Alcohol Studies, 2006

[82] Rehm J. The risks associated with alcohol use and alcoholism. Alcohol Res Health 2011;34(2):135-43.

[83] Bègue L, Perez-Diaz C, Subra B, Ceaux E, Arvers P, Bricout VA, Roché S, Swendsen J, Zorman $\mathrm{M}$. The role of alcohol consumption in female victimization: findings from a French representative sample. Subst Use Misuse 2012;47(1):1-11. 